\title{
Occurrence and detection method evaluation of group B streptococcus from prenatal vaginal specimen in Northwest China
}

Yun Xie ${ }^{1}$, JunLan Yang ${ }^{1}$, Peng Zhao ${ }^{2}$, Hui Jia ${ }^{1}$ and Qi Wang ${ }^{3^{*}}$

\begin{abstract}
Sensitive and efficient detection of Group B Streptococcus (GBS) colonization in pregnant women is essential for prescription of prophylaxis at the time of delivery as GBS is an opportunistic pathogen known to cause infant mortality. In this report, two studies were conducted on the methods of GBS detection in Shaanxi province, China, a region lacking data for GBS detection and occurrence. For Study 1, 100 GBS culture-positive vaginal swabs were collected from 1,567 pregnant women for evaluation by direct latex agglutination test. In Study 2, 200 GBS vaginal swabs were evaluated by three culture methods (sheep blood agar (SBA), Columbia colistin-nalidixic agar (CNA), and selective carrot broth (SCB)) followed by analysis using a latex agglutination test. GBS was detected in $6.4 \%$ of specimens in Study 1 and $10.5 \%$ of specimens in Study 2. The results of the latex agglutination test in both studies were accurate with samples exhibiting high to moderate GBS growth, but the accuracy declined for samples with low GBS growth. The evaluation of culture methods for GBS detection revealed the sensitivity of SCB ( $95.2 \%, p=0.004)$ was significantly higher than that of the SBA medium (57.1\%). The sensitivity reported for SCB (95.2\%) was higher than CNA (76.0\%), but the difference was not statistically significant $(p=0.078)$. These results indicate a selective broth, such as SCB, is ideal for accuracy at low growth levels, but a direct latex agglutination test could be used as an alternative for rapid detection of GBS in circumstances requiring immediate detection.
\end{abstract}

Keywords: Group B streptococcus, Pregnant women, Colonization rate, Carrot Broth, PathoDxtra Strep Grouping Kit, Direct latex agglutination test

\section{Findings}

\section{Introduction}

Group B streptococcus (GBS) is a pathogenic bacterium that causes morbidity and mortality among neonates worldwide [10, 21]. Vertical transmission of GBS from a puerperant to her newborn during delivery can lead to GBS infection resulting in a fatality rate of approximately $50 \%$ [5]. The rate of neonatal GBS infection ranges from 0.17 to 3.06 per 1000 live births in developing countries $[4,8]$.

\footnotetext{
*Correspondence: wangqi_bio@sina.com

${ }^{3}$ Department of Clinical Laboratory, the Second Affiliated Hospital of Xi'an Jiaotong University, Xi'an, Shaanxi 710004, China

Full list of author information is available at the end of the article
}

Studies have shown that GBS screening and intrapartum antibiotic prophylaxis can prevent neonatal transmission of GBS [18]. The sensitivities of detection methods for perinatal GBS colonization are, however, dependent upon specimen delivery time and culture medium [15]. Traditionally, specimens were evaluated using two solid medium, sheep blood agar (SBA) and Columbia colistin-nalidixic agar (CNA), both of which underestimate the incidence of GBS [12]. Therefore, the CDC recommends use of a selective broth medium, such as LIM broth or carrot broth (SCB) [22]. Previous studies revealed the colonization rate of GBS using the SCB culture method was up to $15 \%$ more sensitive than LIM broth $([7,19])$. However, this method is expensive and time-consuming as it takes at least 6 hours to observe a 
positive reaction [2]. Therefore, evaluation of current methods to determine the most sensitive, cost effective and time efficient detection test is beneficial [16]. The aim of this study was to evaluate the occurrence and detection methods of GBS from pregnant women in Shaanxi province, China.

\section{Materials and methods}

\section{Study design}

This research, consisting of two independent studies, was conducted at Northwest Women's and Children's Hospital in Xi'an, China. For Study 1, 1,567 pregnant women at 32-37 weeks of gestation were selected from January 2014-December 2014 for GBS screening by the SBA method followed by a direct latex agglutination test. For Study 2, 200 pregnant women were selected from December 2014-March 2015 for GBS screening by the SBA, CNA and SCB culture methods followed by a latex agglutination test. All individuals that recently used antibiotics, had symptomatic vaginal discharge, or an acute illness were excluded. The Research Ethics Committee of Northwest Women's and Children's Hospital approved the study and gave the approval number (\#3/2014) and informed written consent was obtained for all participants.

\section{SBA, CNA, and SCB methods}

Duplicate vaginal swabs were collected simultaneously from each pregnant woman for both studies using the Copan ESwab Collection and Transport System (Copan Italia, Brescia, Italy). Of the duplicated swabs, one swab was plated on either SBA only (for Study 1) or on either CNA (Becton, Dickinson and Company, Shanghai, China) and SBA followed by culture in SCB (Hardy Diagnostics, Santa Maria, CA) for Study 2. The other swab was stored at $4{ }^{\circ} \mathrm{C}$. All CNA and SBA plates were incubated at $37^{\circ} \mathrm{C}$ and $5 \% \mathrm{CO}_{2}$ for 18 to $48 \mathrm{~h}$ and all SCB cultures were incubated at $35{ }^{\circ} \mathrm{C}$ ambient air for 18 to $48 \mathrm{~h}$. Aliquots of SCB cultures were subsequently plated on SBA. Cultures growing in $\mathrm{SCB}$ were positive if a visible change from colorless to red/orange was observed. Confirmation of GBSlike isolates was achieved by either the CAMP test, latex agglutination test (PathoDxtra Strep Grouping Kit), API 20 STREP system, or Vitek2 compact system prior to an additional latex agglutination test (Fig. 1).

\section{Latex agglutination test method}

Analysis of GBS positive specimens determined by SCB was achieved using $0.5 \mathrm{~mL}$ of culture in a direct latex agglutination test. Alternatively, analysis of GBS positive specimens by the SBA and CNA culture method was achieved using extracts from the corresponding refrigerated swab. The PathoDxtra Strep Grouping Kit (Oxoid) was used to extract and/or test the samples according to manufacturer's instructions. Each latex agglutination test included one negative control swab immersed in sterile broth and one positive control swab dipped in a broth containing group B strain ATCC 12403. The turbidity of the suspension was adjusted to the McFarland 0.5 turbidity standard. All samples were stored at $4{ }^{\circ} \mathrm{C}$.

\section{PCR analysis}

Polymerase chain reaction (PCR) was used to confirm positive growth of GBS from samples in Study 2 by detection of the $c f b$ gene (CAMP factor). PCR primers for

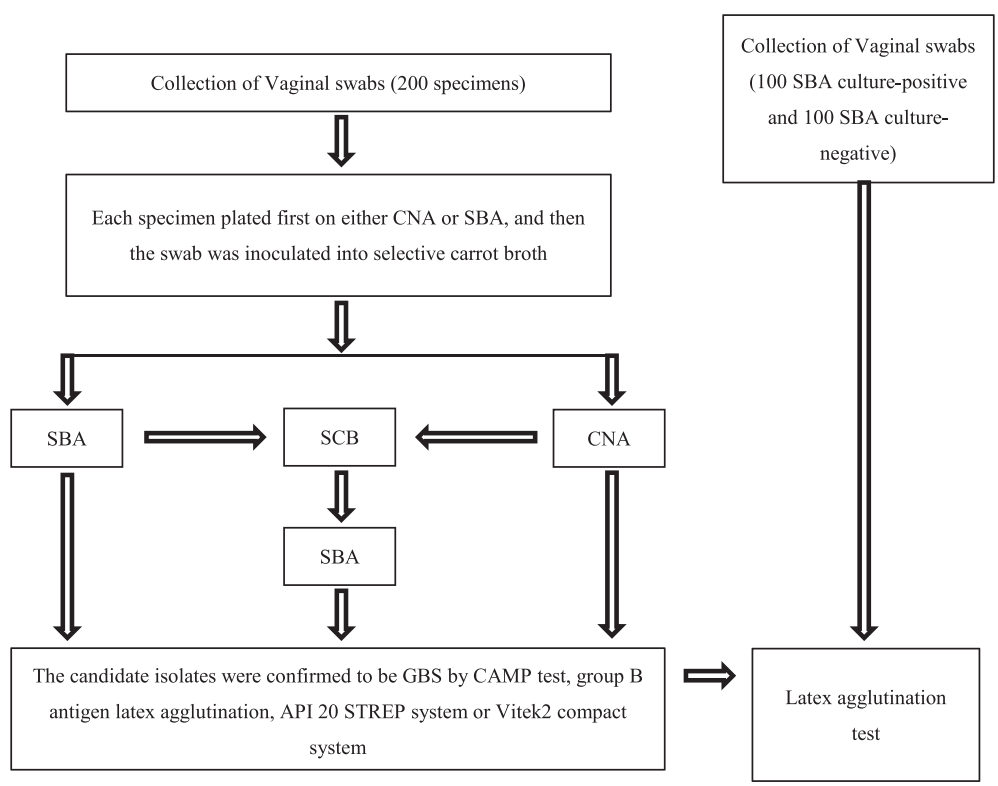

Fig. 1 Laboratory procedure of the vaginal specimens for the isolation and latex agglutination test of GBS 
Table 1 Latex agglutination test results as compared with different density levels of GBS for 100 culture-positive specimens

\begin{tabular}{lllllll}
\hline Density level of GBS (c.f.u. per swab) & $\begin{array}{l}\text { No. of samples } \\
\text { tested positive }(n)\end{array}$ & $\begin{array}{l}\text { No. of samples } \\
\text { positive for GBS }(n)\end{array}$ & Sensitivity (\%) & Specificity (\%) & PPV (\%) & NPV (\%) \\
\hline High (>100) & 22 & 22 & $22 / 22(100 \%)$ & $22 / 22(100 \%)$ & $22 / 22(100 \%)$ & $22 / 22(100 \%)$ \\
Moderate (50-100) & 27 & 27 & $27 / 27(100 \%)$ & $27 / 27(100 \%)$ & $27 / 27(100 \%)$ & $27 / 27(100 \%)$ \\
Low (10-50) & 24 & 36 & $24 / 36(66.7 \%)$ & $36 / 36(100 \%)$ & $24 / 24(100 \%)$ & $36 / 48(75.0 \%)$ \\
Little $(<10)$ & 2 & 15 & $2 / 15(13.3 \%)$ & $15 / 15(100 \%)$ & $2 / 2(100 \%)$ & $15 / 28(53.6 \%)$ \\
\hline
\end{tabular}

cbf were as follows: primer $1\left(5^{\prime}\right.$-ATC GTT ATG GTT TTT ACA TGA-3') and primer 2 (5'-TTA TTT TAA TGC TGT TTG AAG TG-3'). Parameters for PCR amplification were as previously described [1].

\section{Statistical analysis}

The significance test of proportions was used to determine if detection rate differences were significant. The McNemar test for correlated percentages and the $\chi^{2}$ test with Yates' correction were used to analyze qualitative variables. The alpha level was set at 0.05 , in which $p<0.05$ was considered statistically significant.

\section{Results}

Latex agglutination test result of SBA method

In Study 1, the median age of enrolled women was $25.7 \pm 4.8$ years. Out of 1,567 samples analyzed, $6.4 \%$ $(100 / 1,567)$ were positive. The positive vaginal swabs determined by the SBA method were categorized into the following four levels of growth density: high $(>100$ c.f.u. per swab), moderate (50-100 c.f.u. per swab), low (10-50 c.f.u. per swab) and little (<10 c.f.u. per swab). Of the 100 culture-positive samples, $75 \%$ were found to be GBS positive by direct latex agglutination (Table 1). Cultures with heavy or moderate density yielded robust results of $100 \%$ for sensitivity, specificity, positive predictive value (PPV), and negative predictive value (NPV). The sensitivity and negative predictive value declined considerably with density, $66.7 \%$ and $75 \%$ for low density and $13.3 \%$ and $53.6 \%$ for little density, respectively. This was a screen test. It is not necessary to do statistical analysis.

\section{SBA, CNA, and SCB followed by latex agglutination test}

A total of 200 vaginal swab specimens were collected for evaluation of GBS detection by SBA, CNA, and SCB in Study 2. The median age of enrolled women was $24.3 \pm$ 4.6 years. Of the 200 specimens tested, $10.5 \%(21 / 200)$ tested positive by PCR. The sensitivity of the culture media was significantly higher for SCB $(95.2 \%, p=0.004)$ than for the SBA (57.1\%). Despite the higher sensitivity for SCB (95.2\%) compared to CNA (76.0\%), the difference was not statistically significant $(p=0.078)$ (Table 2$)$.
The results of the direct latex agglutination test of positive cultures as determined by the SBA, CNA, and SCB methods (Table 3) revealed that $87.5 \%$ of the samples yielded a positive result by latex method. Positive results by latex agglutination test from each culture method are as follows: SBA $(83.3 \%=9 / 12)$, CNA $(75 \%=12 / 16)$ and SCB $(100 \%=20 / 20)$. Similar to the results of Study 1, the sensitivity, specificity, PPV, and NPV were $100 \%$ for specimens with heavy or moderate growth density. For the low growth cultures, specificity and PPV remained at $100 \%$, but a decline in sensitivity and NPV was detected (Table 3).

Here, we also did the statistical power analysis. All individuals were excluded if they recently used antibiotics and showed symptomatic vaginal discharge or an acute illness. For the evaluation of different culture methods, we conducted statistical analysis by using the $\chi^{2}$ test with Yates' correction with SPSS 19.0 software. The alpha level was set at 0.05 , in which $p<0.05$ was considered statistically significant. The detection rates for the vaginal swabs were $10.0 \%, 8.0 \%$, and $6.0 \%$ on $\mathrm{SCB}$, CNA and SBA, respectively. The GBS isolation rates of SCB and CNA were higher than SBA. However, there were no statistically difference $(p>0.05)$ among them. The sensitivity of the culture media was significantly higher for $\operatorname{SCB}(95.2 \%, p=0.004)$ than for the SBA (57.1\%). Despite the higher sensitivity reported for SCB (95.2\%) compared to CNA (76.0\%) for the vaginal swabs, the difference was not statistically significant $(p=0.078)$. Thus, selective broth SCB provided the highest significant sensitivity for the detection of GBS among the three culture methods.

Table 2 Comparison of results for SBA, CNA, and the selective broth for detection of GBS

\begin{tabular}{|c|c|c|c|}
\hline \multirow[t]{2}{*}{ Culture medium } & \multirow{2}{*}{$\begin{array}{l}\text { GBS positive } \\
\text { samples }(n)\end{array}$} & \multicolumn{2}{|c|}{ Vaginal swabs $(n=200)$} \\
\hline & & $\begin{array}{l}\text { GBS isolation } \\
\text { rate }(\%)\end{array}$ & Sensitivity (\%) \\
\hline SBA & 12 & 6.0 & 57.1 \\
\hline CNA & 16 & 8.0 & 76.0 \\
\hline$S C B$ & 20 & 10.0 & 95.2 \\
\hline True positive GBS cultures & 21 & 10.5 & \\
\hline
\end{tabular}

${ }^{\mathrm{a} S e n s i t i v i t y ~ w a s ~ c a l c u l a t e d ~ i n ~ c o m p a r i s o n ~ t o ~ t r u e ~ p o s i t i v e ~ c u l t u r e s ~ o f ~ a l l ~ m e d i a ~}$ 
Table $\mathbf{3}$ The results of latex agglutination test accompanied with the three culture methods

\begin{tabular}{|c|c|c|c|c|c|c|c|}
\hline Culture medium & $\begin{array}{l}\text { Level of GBS density } \\
\text { (c.f.u. per swab) }\end{array}$ & $\begin{array}{l}\text { No. of samples } \\
\text { tested positive }(n)\end{array}$ & $\begin{array}{l}\text { No. of samples } \\
\text { positive for GBS (n) }\end{array}$ & Sensitivity (\%) & Specificity (\%) & PPV (\%) & NPV (\%) \\
\hline \multirow[t]{4}{*}{ SBA } & High (>100) & 3 & 3 & 100 & 100 & 100 & 100 \\
\hline & Moderate (50-100) & 4 & 4 & 100 & 100 & 100 & 100 \\
\hline & Low (10-50) & 3 & 5 & 60 & 100 & 100 & 71.4 \\
\hline & Little $(<10)$ & 0 & 0 & / & / & / & / \\
\hline \multirow[t]{4}{*}{ CNA } & High (>100) & 3 & 3 & 100 & 100 & 100 & 100 \\
\hline & Moderate (50-100) & 5 & 5 & 100 & 100 & 100 & 100 \\
\hline & Low (10-50) & 4 & 7 & 57.1 & 100 & 100 & 70 \\
\hline & Little $(<10)$ & 0 & 1 & 0 & 100 & 100 & 50 \\
\hline \multirow[t]{4}{*}{ SCB } & High (>100) & 15 & 15 & 100 & 100 & 100 & 100 \\
\hline & Moderate (50-100) & 5 & 5 & 100 & 100 & 100 & 100 \\
\hline & Low (10-50) & 0 & 0 & / & / & / & / \\
\hline & Little $(<10)$ & 0 & 0 & / & / & / & / \\
\hline
\end{tabular}

\section{Discussion}

Research in the area of GBS infections is important for international perinatal medicine as pregnant women can be asymptomatic carriers $[13,14]$. Studies indicate that use of antibiotic prophylaxis contributes to a significant decrease in the incidence of newborn GBS infection in the USA and UK $[11,17]$. However, there are few reports on the detection methods for the identification of GBS in China.

We evaluated three culture mediums for the identification of GBS. Comparison of SBA, CNA and SCB methods revealed that $\mathrm{SCB}$ was the most reliable. The sensitivity of CNA was similar to previous reports $[6,9]$ as was the sensitivity of SCB [3]. SCB is more expensive than SBA or CNA but processing time is reduced. Our results provide support for the current recommendations proposed by CDC for GBS detection in pregnant women by the SCB method.

In addition, we investigated a direct latex method for rapid detection of GBS. Sensitivity and specificity of the latex agglutination test were previously reported as $85.7 \%$ and $99.3 \%$ [20]. In our study, we found the latex agglutination test results were dependent on the density of GBS colonization. We determined a GBS density greater than 50 c.f.u. tested positive by the latex method while below 50 c.f.u. resulted in a significant decrease in the sensitivity and negative predictive values. The advantage of using the latex agglutination method over the culture methods was ease of use and a reduction in turnaround time. Therefore, the latex method is very likely beneficial for pregnant women who are prone premature labor and require immediate GBS detection.

The study herein provides data on the effectiveness of detection methods and occurrences of GBS in China. We found that SCB provided the highest sensitivity for the detection of GBS among the three culture methods tested; however, the use of a direct latex agglutination method is an alternative option with reduced labor costs and turnaround time that can be implemented in critical situations.

\section{Abbreviations}

GBS: Group B Streptococcus; SCB: Selective carrot broth; CNA: Columbia colistin-nalidixic agar; SBA: Sheep blood agar; PCR: Polymerase chain reaction; PPV: Positive predictive value; NPV: Negative predictive value.

Competing interests

The authors declare that they have no competing interests.

\section{Authors' contributions}

YX carried out all the experimental design and participated in the analysis and drafted the manuscript. JY helped the data analysis. PZ participated in the experimental design. $\mathrm{HJ}$ performed the data analysis. QW conceived of the study and participated in its design and coordination and helped to draft the manuscript. All authors read and approved the final manuscript.

\section{Acknowledgments}

The authors express their sincere gratitude to Ling Dong and Shuang Ma for excellent technical assistance.

\section{Author details}

'Department of Clinical Microbiology, Medical Laboratory Centre, Northwest Women's and Children's Hospital, Xi'an, Shaanxi 710061, China. ${ }^{2}$ Colleges of Life Sciences, Northwest University, Xi'an, Shaanxi 710069, China.

${ }^{3}$ Department of Clinical Laboratory, the Second Affiliated Hospital of Xi'an Jiaotong University, Xi'an, Shaanxi 710004, China.

Received: 3 November 2015 Accepted: 14 January 2016

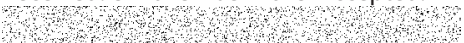

\section{References}

1. Abdulmawjood A, Lämmler C. Amplification of $16 \mathrm{~S}$ ribosomal RNA gene sequences for the identification of streptococci of Lancefield group B. Res Vet Sci. 1999;67:159-62.

2. Block T, Munson E, Culver A, Vaughan K, Hryciuk JE. Comparison of carrot broth- and selective Todd-Hewitt broth-enhanced PCR protocols for real-time detection of Streptococcus agalactiae in prenatal vaginal/anorectal specimens. J Clin Microbiol. 2008;46:3615-20. doi:10.1128/jcm.01262-08.

3. Church DL, Baxter H, Lloyd T, Miller B, Elsayed S. Evaluation of StrepB carrot broth versus Lim broth for detection of group B Streptococcus colonization status of near-term pregnant women. J Clin Microbiol. 2008;46:2780-2. doi:10.1128/jcm.00557-08. 
4. Dagnew AF, Cunnington MC, Dube Q, Edwards MS, French N, Heyderman $\mathrm{RS}$, et al. Variationin reported neonatal group B streptococcal disease incidence in developing countries. Clin Infect Dis. 2012;55:91-102. doi:10.1093/cid/cis395.

5. Edmond KM, Kortsalioudaki C, Scott S, Schrag SJ, Zaidi AK, Cousens S, et al. Group B streptococcal disease in infants aged younger than 3 months: systematic review and meta-analysis. Lancet. 2012;379:547-56. doi:10.1016/50140-6736(11)61651-6.

6. Ghaddar N, Alfouzan W, Anastasiadis E, A Jiser T, Itani SE, Dernaika R, et al. Evaluation of chromogenic medium and direct latex agglutination test for detection of group B streptococcus in vaginal specimens from pregnant women in Lebanon and Kuwait. J Med Microbiol. 2014;63:1395-9. doi:10.1099/jmm.0.066738-0.

7. Hutchens KA, Schreckenberger PC. Comparison of real-time PCR (Cepheid Smart Cycler) with standard LIM broth culture and Strep B Carrot Broth ${ }^{\text {TM }}$ for the detection of group B Streptococcus in pre-natalvaginal/rectal specimens, abstr. C-138, p. 124-125. 2006. Abstr. 106th Gen. Meet. Am. Soc. Microbiol. American Society for Microbiology, Washington, DC.

8. Kuruvilla KA, Thomas N, Jesudasan MV, Jana AK. Neonatal group B streptococcal bacteraemia in India: ten years' experience. Acta Paediatr. 1999:88:1031-2.

9. Kwatra G, Madhi SA, Cutland CL, Buchmann EJ, Adrian PV. Evaluation of Trans-Vag broth, colistin-nalidixic agar, and CHROM agar StrepB for detection of group B Streptococcus in vaginal and rectal swabs from pregnant women in South Africa. J Clin Microbiol. 2013;51:2515-9. doi:10.1128/jcm.00251-13.

10. Lu B, Li D, Cui Y, Sui W, Huang L, Lu X. Epidemiology of Group B streptococcus isolated from pregnant women in Beijing, China. Clin Microbiol Infect. 2014;20:370-3.

11. Neugut Al, Ghatak AT, Miller RL. Anaphylaxis in the united states: an investigation into its epidemiology. Arch Intern Med. 2001;161:15-21.

12. Overman $S B$, Eley DD, Jacobs BE, Ribes JA. Evaluation of methods to increase the sensitivity and timeliness of detection of Streptococcus agalactiae in pregnant women. J Clin Microbiol. 2002;40:4329-31.

13. Otaguiri ES, Morguette AE, Tavares ER, dos Santos PM, Morey AT, Cardoso JD, et al. Commensal Streptococcus agalactiae isolated from patients seen at University Hospital of Londrina, Paraná, Brazil: capsular types, genotyping, antimicrobial susceptibility and virulence determinants. BMC Microbiol. 2013;13:297. doi:10.1186/1471-2180-13-297.

14. Phares CR, Lynfield R, Farley MM, Mohle-Boetani J, Harrison LH, Petit S, et al. Epidemiology of invasive group B streptococcal disease in the United States, 1999-2005. JAMA. 2008;299:2056-65.

15. Quinlan JD, Hill DA, Maxwell BD, Boone S, Hoover F, Lense JJ. The necessity of both anorectal and vaginal cultures for group B streptococcus screening during pregnancy. J Fam Pract. 2000;49:447-8.

16. Rallu F, Barriga P, Scrivo C, Martel-Laferrière V, Laferrière C. Sensitivities of antigen detection and PCR assays greatly increased compared to that of the standard culture method for screening for group B streptococcus carriage in pregnant women. J Clin Microbiol. 2006;44:725-8.

17. Royal College of Obstetricians and Gynaecologists (RCOG). Prevention of Early-onset Neonatal Group B Streptococcal Disease. In: Green-top Guideline Volume 36. London: RCOG; 2003

18. Scasso S, Laufer J, Rodriguez G, Alonso JG, Sosa CG. Vaginal group B streptococcus status during intrapartum antibiotic prophylaxis. Int J GynaecolObstet. 2015;129:9-12. doi:10.1016/j.jigo.2014.10.018.

19. Schrecken berger P, Hsiung AY, Marnell C, Alonso JG.2005. Evaluation of Strep B Carrot Broth ${ }^{\text {TM }}$ and LIM broth methods for recovery of group B streptococci (GBS). Results of a multi-center trial, abstr. C-109, p. 124. Abstr. 105th Gen. Meet. Am. Soc. Micro biol. American Society for Microbiology, Washington, DC

20. Wald ER, Dashefsky B, Green M, Harger J, Parise M, Korey C, et al. Rapid detection of group B streptococci directly from vaginal swabs. J Clin Microbiol. 1987;25:573-4.

21. Verani JR, Schrag SJ. Group B streptococcal disease in infants: progress in prevention and continued challenges. Clin Perinatol. 2010;37:375-92. doi:10.1016/j.clp.2010.02.002.

22. Verani JR, McGee L, Schrag SJ. Prevention of perinatal group B streptococcal disease-revised guidelines from CDC, 2010. MMWR Recomm Rep. 2010;59(RR-10):1-36.

\section{Submit your next manuscript to BioMed Central and we will help you at every step:}

- We accept pre-submission inquiries

- Our selector tool helps you to find the most relevant journal

- We provide round the clock customer support

- Convenient online submission

- Thorough peer review

- Inclusion in PubMed and all major indexing services

- Maximum visibility for your research

Submit your manuscript at www.biomedcentral.com/submit

C Biomed Central 